\section{Magenkarzinom: Taxan in der Neoadjuvanz - Potenzial bisher nicht ausgelotet}

\author{
Die adjuvante Fluorouracil-basierte Chemotherapie (CT) nach kurativer \\ Resektion des lokal fortgeschrittenen Magenkarzinoms gilt als Standard. \\ Neoadjuvante Regimes mit Taxanen könnten Vorteile haben - wenngleich \\ diese noch nicht evidenzbasiert nachgewiesen sind.
}

Dis ie Fluorouracil-basierte adjuvante Chemotherapie bei therapienaiven Patienten mit resektablem Magenkarzinom hat gegenüber einer alleinigen Operation leichte Vorteile, so das Ergebnis von Metaanalysen. Möglicherweise haben Patienten mit resektablem Magenkarzinom unter perioperativen Therapieregimes bessere Überlebenschancen. Um weitere Einsichten in das Potenzial einer neoadjuvanten Behandlung zu erhalten, wurde unter Federführung der Swiss Group for Clinical Cancer Research (SAKK) und des European Institute of Oncology (IEO) 1999 eine europäische Phase-III-Studie aufgelegt, in der eine neoadjuvante im Vergleich zu einer adjuvanten Behandlung mit Docetaxel, Cisplatin und 5-Fluorouracil (TCF) bei Patienten mit resektablem Magenkarzinom untersucht werden sollte. Aufgrund der nur schleppenden Rekrutierung wurde die Studie allerdings vorzeitig abgebrochen.

Die 10-Jahres-Daten der 69 randomisierten Patienten mit lokal fortgeschrittenem resektablem Magenkarzinom wurden jetzt analysiert. Die Kohorte hatte in einer 1:1-Randomisierung entweder die neoadjuvante oder die adjuvante CT erhalten. Weder beim primären Studienendpunkt ereignisfreies
Überleben (EFS) noch beim sekundären Endpunkt Gesamtüberleben (OS) ergaben sich signifikante Unterschiede zwischen neoadjuvanter und adjuvanterTherapie: Das EFS betrug in beiden Armen 2,5 Jahre, das OS unter Neoadjuvanz 4,3 und unter Adjuvanz 3,7 Jahre. Die Neoadjuvanz erlaubte eine höhere Dosisintensität und hatte gleichzeitig weniger Nebenwirkungen, die Operation ließ sich sicher durchführen.

Fazit: Das TCF-Regime, neoadjuvant beim resektablen Magenkarzinom eingesetzt, scheint im Vergleich zum adjuvanten Einsatz Vorteile hinsichtlich der Dosisintensität und den Nebenwirkungen zu haben. Inwieweit sich durch die Neoadjuvanz Vorteile beim Gesamtüberleben ergeben, sollte jetzt überprüft werden.

Barbara Kreutzkamp

Fazio $\mathrm{N}$ et al. Preoperative versus postoperative docetaxel-cisplatin-fluorouracil (TCF) chemotherapy in locally advanced resectable gastric carcinoma: 10-year follow-up of the SAKK 43/99 phase III trial. Ann Oncol. 2016;27(4):668-73.

\title{
Operation vor der Chemotherapie bei fortgeschrittenem Magenkarzinom?
}

Die Prognose für Patienten mit Magenkarzinom und hepatischen, peritonealen oder entfernteren Lymphknotenmetastasen ist schlecht. Asiatische Kollegen gingen der Frage nach, ob das Überleben durch eine Gastrektomie vor der Chemotherapie verbessert werden kann.

A n der Studie nahmen Magenkarzinompatienten im Alter von 20-75 Jahren teil, die einen Faktor für Nichtheilbarkeit aufwiesen: entweder Lebermetastasen, peritoneale Metastasen oder paraaortale Lymphknotenmetastasen. Randomisiert erhielten sie entweder eine Chemotherapie alleine oder eine Gastrektomie mit D1-Lymphadenektomie, aber ohne Metastasenresektion, mit nachfolgender Chemotherapie (orales $\mathrm{S}-1,80 \mathrm{mg} / \mathrm{m}^{2}$ an den Tagen $1-21$, und Cisplatin, $60 \mathrm{mg} / \mathrm{m}^{2}$ an Tag 8) in 5-Wochen-Zyklen. Der primäre Endpunkt der Studie war das Gesamtüberleben in der Intention-to-Treat-Population.

In der ersten Interimsanalyse war die Wahrscheinlichkeit, dass die kombinierte Therapie der alleinigen Chemothera- pie überlegen war, mit 13,2 \% gering. Daraufhin wurde die Studie zu diesem Zeitpunkt mit 175 Teilnehmern geschlossen. Die Gesamtüberlebensrate nach 2 Jahren lag für die Patienten mit Chemotherapie alleine $(\mathrm{n}=86)$ bei $31,7 \%$ (95\%-Konfidenzintervall [95\%-KI] 21,7-42,2), bei kombinierter Therapie $(\mathrm{n}=89)$ bei $25,1 \%$ (95\%-KI 16,2-34,9). Das mediane Gesamtüberleben in den beiden Gruppen betrug 16,6 Monate (95\%-KI 13,7-19,8) bzw. 14,3 Monate (11,8-16,3; Hazard Ratio [HR] 1,09; $95 \%-\mathrm{KI} \quad 0,78-1,52$; $\mathrm{p}=0,70)$.

In der Gruppe mit Gastrektomie und Chemotherapie verzeichneten die Untersucher eine höhere Inzidenz von chemotherapiebedingten Nebenwirkungen des Grads 3/4 als in der Gruppe mit alleini- ger Chemotherapie, z. B. häufiger eine Leukopenie (18 vs. $3 \%$ ), Anorexie (29 vs. $12 \%)$, Nausea (15 vs. $5 \%$ ) und Hyponatriämie (9 vs. $5 \%$ ). Ein Patient der Chemotherapiegruppe verstarb therapiebedingt infolge eines plötzlichen Herzstillstands, ein Patient der Kombinationsgruppe verstarb aufgrund eines raschen Progresses der peritonealen Metastasierung 12 Tage nach Operation.

Fazit: Eine Gastrektomie mit nachfolgender Chemotherapie zeigt auch bei $\mathrm{Pa}$ tienten mit Magenkarzinom und einem einzelnen Faktor für Nicht-Heilbarkeit keinerlei Überlebensvorteil gegenüber einer Chemotherapie alleine. Damit kann die Gastrektomie in dieser Situation nicht empfohlen werden.

Friederike Klein

Fujitani K et al. Gastrectomy plus chemotherapy versus chemotherapy alone for advanced gastric cancer with a single non-curable factor (REGATTA): a phase 3, randomised controlled trial. Lancet Oncol. 2016;17(3):309-18. 